\title{
An Inventory Model for Decaying Items, Considering Multi Variate Consumption Rate with Partial Backlogging
}

\author{
Sanjay Sharma ${ }^{1 *}$ and S. R. Singh ${ }^{2}$ \\ 1Research Scholar GEU, Dehradun, India; sanjaysharma698@gmail.com \\ 2Department of Mathematics, D.N. College, Meerut; shivrajpundir@gmail.com
}

\begin{abstract}
In most of the research articles, demand is considered either constant or a function of single variable which is not practical; customers' purchasing deeds may be affected by factors such as selling price and inventory level consequently in the present study we have considered demand rate as a function of stock-level and selling price both. Various researchers considered the situation in which shortages are either completely backlogged or completely lost which is not realistic therefore in development of this model it is assumed that shortages are partially backlogged and the most realistic backlogging rate is considered. In this research, we proposed a partial backlogging inventory model for decaying items considering stock and selling price dependent demand rate in fuzzy environment. In developing the model demand rate, ordering cost, purchasing cost, holding cost, backordering cost and opportunity cost are considered as triangular fuzzy numbers. Graded mean integration representation method is used for defuzzification. A numerical example is provided to illustrate the problem. Sensitivity analysis of the optimal solution with respect to the changes in the value of system parameters is also discussed.
\end{abstract}

Keywords: Inventory Model, Triangular Fuzzy Numbers, Signed Distance, Stock and Price Dependent Demand.

\section{Introduction}

Many realistic experiences disclose that some but not all customers will wait for backlogged items during a shortage period, such as for fashionable supplies and the products with short life cycle. The longer the waiting time is, the lesser the backlogging rate would be. According to such phenomenon, backlogging rate should not be disregarded. Thus it is necessary to consider backlogging rate. Researchers, such as Park [8], Hollier and Mak [9] and Wee [11] developed inventory models with partial backorders. Goyal and Giri [10] developed production inventory model with shortages partially backlogged. Wu et al. [12] developed a replenishment policy for deteriorating items with stock-dependent demand and partial backlogging. Singh [17] presented a perishable inventory model with quadratic demand and partial backlogging. Skouri et al. [14] presented an inventory model with ramp type demand rate, partial backlogging and Weibull deterioration rate.

Deterioration is a natural phenomenon in many real situations so it can plays an important role in developing an inventory model. Generally, deterioration is defined as damage, spoilage, decay and obsolescence, vaporization,

*Corresponding author:

Sanjay Sharma (sanjaysharma698@gmail.com) 
etc., that result in decrease of value of the original one. In most of the classical problems deterioration is considered. The first model for decaying items was presented by Ghare and Schrader (21). It was extended by Covert and Philip (22) considering Weibull distribution deterioration. Goswami et al. [1] developed an inventory model for deteriorating items with shortages and a linear trend in demand also a complete survey for deteriorating inventory models was presented by Raafat (23). Some other papers relevant to this topic are Teng et al., (27), Chang et al. [13] and Abdul and Murata, (24).

Many classical research articles assumed that the demand is constant during the sales period. In real life, the demand may be inspired if there is a large pile of goods displayed on shelf. Levin et al. (6) termed that the more goods displayed on shelf, the more customer's demand will be generated. Gupta and Vrat (26) were the first to build up models for stock-dependent consumption rate. Baker and Urban [2] established an EOQ model for a powerform inventory-level-dependent demand pattern. Mandal and Phaujdar [3] then developed a production inventory model for deteriorating items with uniform rate of production and linearly stock-dependent demand. Other papers related to this research area are $[25,4,5,7,15,16]$.

In real life situations, due to impreciseness of parameters in inventory, it is important to consider them as fuzzy numbers. The concept of fuzzy set theory first introduced by Zadeh L. [18], after that Park [19] extended the classical EOQ model by introducing the fuzziness of ordering cost and holding cost. A fuzzy model for inventory with backorder, where the backorder quantity was fuzzified as the triangular fuzzy number was presented by Chang et al. (28). Recently a supply chain inventory model under fuzzy demand was established by Ruoning Xu, Xiaoyan Zhai [20].

Above cited papers reveals that many research articles are developed in which demand is considered as the function of stock level or selling price, shortages are allowed and partially backlogged, but there is no such research paper which is partially backlogged assuming demand rate as the function of selling price and inventory level in fuzzy environment. In lots of business practices it is observed that several parameters in inventory system are imprecise. Therefore, it is necessary to consider them as fuzzy numbers while developing the inventory model.

In the present study we have developed a partial backlogging inventory model for deteriorating items considering stock and price sensitive demand rate in fuzzy surroundings. A numerical example to prove that the optimal solution exists and is unique is provided and the sensitivity analysis with respect to system parameters is discussed. The concavity is also shown through the figure 2.

\section{Assumptions and Notations}

The basic assumptions of the model (figure 1) are as follows:

(1) The demand rate is a function of stock and selling price considered as $f(t)=(a+b Q(t)-p)$ where $a>0,0$ $<b<1, a>b$ and $p$ is selling price.

(2) Holding cost $h(t)$ per item per time-unit is time dependent and is assumed to be $h(t)=h+\delta t$ where $\delta>0, h>0$.

(3) Shortages are allowed and partially backlogged and rate is assumed to be $1 /(1+\eta t)$ which is a decreasing function of time.

(4) The deterioration rate is time dependent.

(5) $T$ is the length of the cycle.

(6) Replenishment is instantaneous and lead time is zero.

(7) The order quantity in one cycle is $Q$.

(8) The selling price per unit item is $p$.

(9) $A$ is the cost of placing an order.

(10) $c_{1}$ is the purchasing cost per unit per unit.

(11) $c_{2}$ the backorder cost per unit per unit time.

(12) $c_{3}$ the opportunity cost (i.e., goodwill cost) per unit.

(13) $P(T, \mathrm{t}, \mathrm{p})$ the total profit per unit time.

(14) The deterioration of units follows the two parameter Weibull distribution (say) $\theta(\mathrm{t})=\alpha \beta \mathrm{t}^{\beta-1}$ where 0 $<\alpha<1$ is the scale parameter and $\beta>0$ is the shape parameter.

(15) During time $t_{1}$, inventory is depleted due to deterioration and demand of the item. At time $t_{1}$ the inventory becomes zero and shortages start going on.

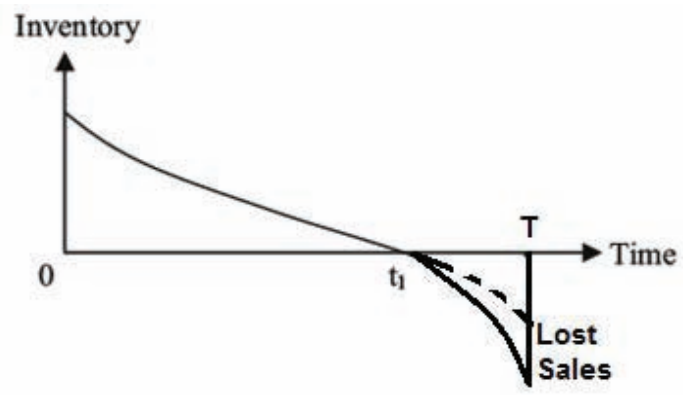

Figure 1. Graphical representation of the model. 


\section{Mathematical Formulation and Solution}

Let $Q(t)$ be the inventory level at time $t(0 \leq t \leq T)$. During the time interval $\left[0, t_{1}\right]$ inventory level decreases due to the combined effect of demand and deterioration both and at $t_{1}$ inventory level depletes up to zero. The differential equation to describe immediate state over $\left[0, t_{1}\right]$ is given by:

$$
Q^{\prime}(t)+a \beta t^{\beta-1} Q(t)=-(a+b Q(t)-p) 0 \leq t \leq t_{1}
$$

Again, during time interval $\left[t_{1}, T\right]$ shortages stars occurring and at $T$ there are maximum shortages, due to partial backordering some sales are lost. The differential equation to describe instant state over $\left[t_{1}, T\right]$ is given by:

$$
Q^{\prime}(t)=-\frac{(a-p)}{[1+\eta(T-t)]} t_{1} \leq t \leq T
$$

With condition $Q\left(t_{1}\right)=0$

Solving equation (1) and equation (2) and neglecting higher powers of $\alpha$, we get

$$
\begin{gathered}
Q(t)=(a-p)\left[t_{1}-t+\frac{b}{2}\left(t_{1}^{2}-t^{2}\right)+\frac{\alpha}{(\beta+1)}\left(t_{1}^{\beta+1}-t^{\beta+1}\right)\right] e^{-\mathrm{bt}-\alpha t^{\beta}} \\
0 \leq t \leq t_{1}
\end{gathered}
$$

and

$$
\begin{gathered}
Q(t)=\frac{(a-p)}{\eta}\left[\log \{1+\eta(T-t)\}-\log \left\{1+\eta\left(T-t_{1}\right)\right\}\right] \\
t_{1} \leq t \leq T
\end{gathered}
$$

At time 0 inventory level is $Q(0)$ and is given by

$$
Q(0)=(a-p)\left(t_{1}+\frac{b t_{1}^{2}}{2}+\frac{\alpha t_{1}^{\beta+1}}{\beta+1}\right)
$$

At time $T$ maximum shortages $\left(Q_{1}\right)$ occurs and is given by

$$
Q_{1}=\frac{(a-p)}{\eta}\left[\log \left\{1+\eta\left(T-t_{1}\right)\right\}\right]
$$

The order quantity is $Q$ and is given by

$$
Q=(a-p)\left(t_{1}+\frac{b t_{1}^{2}}{2}+\frac{\alpha t_{1}^{\beta+1}}{\beta+1}+\frac{1}{\eta} \log \left(1+\eta\left(T-t_{1}\right)\right)\right)
$$

The purchasing cost is

$$
P C=c_{1}(a-p)\left(t_{1}+\frac{b t_{1}^{2}}{2}+\frac{\alpha t_{1}^{\beta+1}}{\beta+1}+\frac{1}{\eta} \log \left(1+\eta\left(T-t_{1}\right)\right)\right)
$$

Ordering cost is

$$
O C=A
$$

\section{Holding cost is}

$$
\begin{aligned}
I H C & =\int_{0}^{t}(h+\delta t) Q(t) d t \\
& =\int_{0}^{t^{t}}(h+\delta t)(a-p)\left[t_{1}-t+\frac{b}{2}\left(t_{1}^{2}-t^{2}\right)+\frac{\alpha}{(\beta+1)}\left(t_{1}^{\beta+1}-t^{\beta+1}\right)\right] e^{-\mathrm{bt}-\alpha t^{\beta}} d t \\
& =(a-p)\left[\begin{array}{l}
\frac{h t_{1}^{2}}{2}+\frac{\delta t_{1}^{3}}{6}+\frac{b h t_{1}^{3}}{6}+\frac{b \delta t_{1}^{4}}{24}-\frac{b^{2} h t_{1}^{4}}{8}-\frac{b^{2} \delta t_{1}^{5}}{15}+\frac{\alpha h t_{1}^{\beta+2}}{\beta+2}-\frac{\alpha h t_{1}^{\beta+2}}{(\beta+1)(\beta+2)} \\
+\frac{3 \alpha t_{1}^{\beta+3}}{2(\beta+3)}-\frac{\alpha b h t_{1}^{\beta+3}}{2(\beta+1)}-\frac{\alpha \delta t_{1}^{\beta+3}}{(\beta+2)}+\frac{\alpha \delta b t_{1}^{\beta+4}}{6(\beta+4)}-\frac{\alpha \delta b t_{1}^{\beta+4}}{2(\beta+2)}-\frac{\alpha^{2} h t_{1}^{2 \beta+2}}{2(\beta+1)^{2}} \\
-\frac{\alpha^{2} \delta t_{1}^{2 \beta+3}}{(\beta+2)(2 \beta+3)}
\end{array}\right.
\end{aligned}
$$

Shortage cost due to backordered is:

$$
\begin{aligned}
B C & =c_{2} \int_{t_{1}}^{T}[-Q(t)] d t \\
& =c_{2} \int_{t_{1}}^{T} \frac{(a-p)}{\eta}\left[\log \{1+\eta(T-t)\}-\log \left\{1+\eta\left(T-t_{1}\right)\right\}\right] d t \\
& =\frac{c_{2}(a-p)}{\eta^{2}}\left[\eta\left(T-t_{1}\right)-\log \left\{1+\eta\left(T-t_{1}\right)\right\}\right]
\end{aligned}
$$

Lost sales cost due to lost sales is:

$$
\begin{aligned}
L S & =c_{3}(a-p) \int_{t_{1}}^{T}\left[1-\frac{1}{(1+\eta(T-t))}\right] d t \\
& =\frac{c_{3}(a-p)}{\eta}\left[\eta\left(T-t_{1}\right)-\log \left\{1+\eta\left(T-t_{1}\right)\right\}\right]
\end{aligned}
$$

Sales revenue is given by:

$$
\begin{aligned}
S R & =p \int_{0}^{t_{1}}(a+b Q(t)-p) d t+p \int_{t_{1}}^{T}(a-p) d t \\
& =p(a-p) T+b(a-p) p\left(\frac{t_{1}^{2}}{2}+\frac{b t_{1}^{3}}{3}+\frac{\alpha t_{1}^{\beta+2}}{\beta+2}\right)
\end{aligned}
$$


From (6), (7), (8), (9) and (10) total profit per unit time is given by:

$$
\begin{aligned}
& P\left(T, t_{1}, p\right)=\frac{1}{T}(S R-O C-P C-I H C-B C-L S) \\
& =\frac{1}{T}\left[\begin{array}{l}
p(a-p) T+b(a-p) p\left(\frac{t_{1}^{2}}{2}+\frac{b t_{1}^{3}}{3}+\frac{\alpha t_{1}^{\beta+2}}{\beta+2}\right)-A-c_{1}(a-p)\left(t_{1}+\frac{b t_{1}^{2}}{2}+\frac{\alpha t_{1}^{\beta+1}}{\beta+1}+\frac{1}{\eta} \log \left(1+\eta\left(T-t_{1}\right)\right)\right) \\
-\frac{c_{2}(a-p)}{\eta^{2}}\left\{\eta\left(T-t_{1}\right)-\log \left(1+\eta\left(T-t_{1}\right)\right)\right\}-\frac{c_{3}(a-p)}{\eta}\left\{\eta\left(T-t_{1}\right)-\log \left(1+\eta\left(T-t_{1}\right)\right)\right\} \\
-(a-p)\left\{\begin{array}{l}
\frac{h t_{1}^{2}}{2}+\frac{\delta t_{1}^{3}}{6}+\frac{b h t_{1}^{3}}{6}+\frac{b \delta t_{1}^{4}}{24}-\frac{b^{2} h t_{1}^{4}}{8}-\frac{b^{2} \delta t_{1}^{5}}{15}+\frac{\alpha h t_{1}^{\beta+2}}{\beta+2}-\frac{\alpha h t_{1}^{\beta+2}}{(\beta+1)(\beta+2)} \\
+\frac{\alpha t_{1}^{\beta+3}}{2(\beta+3)}-\frac{\alpha b h t_{1}^{\beta+3}}{2(\beta+1)}-\frac{\alpha \delta t_{1}^{\beta+3}}{(\beta+2)}+\frac{\alpha \delta b t_{1}^{\beta+4}}{6(\beta+4)}-\frac{\alpha \delta b t_{1}^{\beta+4}}{2(\beta+2)}-\frac{\alpha^{2} h t_{1}^{2 \beta+2}}{2(\beta+1)^{2}} \\
-\frac{\alpha^{2} \delta t_{1}^{2 \beta+3}}{(\beta+2)(2 \beta+3)}
\end{array}\right\}
\end{array}\right]
\end{aligned}
$$

Let $t_{1}=\gamma T, 0<\gamma<1$

Hence, we get the profit function

$$
P(T, p)=\frac{1}{T}\left[\begin{array}{l}
p(a-p) T+b(a-p) p\left(\frac{\gamma^{2} T^{2}}{2}+\frac{b \gamma^{3} T^{3}}{3}+\frac{\alpha \gamma^{\beta+2} T^{\beta+2}}{\beta+2}\right)-A \\
-c_{1}(a-p)\left(\begin{array}{l}
\left.\gamma T+\frac{b \gamma^{2} T^{2}}{2}+\frac{\alpha \gamma^{\beta+1} T^{\beta+1}}{\beta+1}\right) \\
+\frac{1}{\eta} \log (1+\eta(T-\gamma T))
\end{array}\right)-\frac{c_{2}(a-p)}{\eta^{2}}\{\eta(T-\gamma T)-\log (1+\eta(T-\gamma T))\} \\
-\frac{c_{3}(a-p)}{\eta}\{\eta(T-\gamma T)-\log (1+\eta(T-\gamma T))\} \\
-(a-p)\left\{\begin{array}{l}
\frac{h \gamma^{2} T^{2}}{2}+\frac{\delta \gamma^{3} T^{3}}{6}+\frac{b h \gamma^{3} T^{3}}{6}+\frac{b \delta \gamma^{4} T^{4}}{24}-\frac{b^{2} h \gamma^{4} T^{4}}{8}-\frac{b^{2} \delta \gamma^{5} T^{5}}{15}+\frac{\alpha h \gamma^{\beta+2} T^{\beta+2}}{\beta+2} \\
-\frac{\alpha h \gamma^{\beta+2} T^{\beta+2}}{(\beta+1)(\beta+2)}+\frac{3 \alpha \delta \gamma^{\beta+3} T^{\beta+3}}{2(\beta+3)}-\frac{\alpha b h \gamma^{\beta+3} T^{\beta+3}}{2(\beta+1)}-\frac{\alpha \delta \gamma^{\beta+3} T^{\beta+3}}{(\beta+2)}+\frac{\alpha \delta b \gamma^{\beta+4} T^{\beta+4}}{6(\beta+4)} \\
-\frac{\alpha \delta b \gamma^{\beta+4} T^{\beta+4}}{2(\beta+2)}-\frac{\alpha^{2} h \gamma^{2 \beta+2} T^{2 \beta+2}}{2(\beta+1)^{2}}-\frac{\alpha^{2} \delta \gamma^{2 \beta+3} T^{2 \beta+3}}{(\beta+2)(2 \beta+3)}
\end{array}\right.
\end{array}\right\}
$$

Our objective is to maximize the profit function $P(T, p)$. The necessary conditions for maximizing the profit are

We get,

$$
\frac{\partial P(T, p)}{\partial T}=0 \text { and } \frac{\partial P(T, p)}{\partial p}=0
$$




$$
\frac{\partial P(T, p)}{\partial T}=\left[\begin{array}{l}
b(a-p) p\left(\frac{\gamma^{2}}{2}+\frac{2 b \gamma^{3} T}{3}+\frac{a(\beta+1) \gamma^{\beta+2} T^{\beta}}{\beta+2}\right)+\frac{A}{T^{2}}+(a-p)\left(\frac{c_{2}}{\eta^{2}}+\frac{c_{3}}{\eta}\right)\left\{\begin{array}{l}
+\frac{\eta(1-\gamma)}{T(1+\eta(T-\gamma T))} \\
-\frac{1}{T^{2}} \log (1+\eta(T-\gamma T))
\end{array}\right] \\
-c_{1}(a-p)\left(\begin{array}{l}
\left.\frac{b \gamma^{2}}{2}+\frac{a \beta \gamma^{\beta+1} T^{\beta-1}}{\beta+1}+\frac{(1-\gamma)}{T(1+\eta(T-\gamma T))}\right) \\
-\frac{1}{\eta T^{2}} \log (1+\eta(T-\gamma T))
\end{array}\right)\left[\begin{array}{l}
\frac{h \gamma^{2}}{2}+\frac{\delta \gamma^{3} T}{3}+\frac{b h \gamma^{3} T}{3}+\frac{b \delta \gamma^{4} T^{2}}{8}-\frac{3 b^{2} h \gamma^{4} T^{2}}{8}-\frac{4 b^{2} \delta \gamma^{5} T^{3}}{15}+\frac{a h(\beta+1) \gamma^{\beta+2} T^{\beta}}{\beta+2} \\
-\frac{a h \gamma^{\beta+2} T^{\beta}}{(\beta+2)}+\frac{3 a \delta(\beta+2) \gamma^{\beta+3} T^{\beta+1}}{2(\beta+3)}-\alpha \delta \gamma^{\beta+3} T^{\beta+1}-\frac{a b h(\beta+2) \gamma^{\beta+3} T^{\beta+1}}{2(\beta+1)} \\
-(a-p) \\
+\frac{a \delta b(\beta+3) \gamma^{\beta+4} T^{\beta+2}}{6(\beta+4)}-\frac{a \delta b(\beta+3) \gamma^{\beta+4} T^{\beta+2}}{2(\beta+2)}-\frac{a^{2} h(2 \beta+1) \gamma^{2 \beta+2} T^{2 \beta}}{2(\beta+1)^{2}} \\
-\frac{a^{2} \delta(2 \beta+2) \gamma^{2 \beta+3} T^{2 \beta+1}}{(\beta+2)(2 \beta+3)}
\end{array}\right.
\end{array}\right\}=0
$$

and

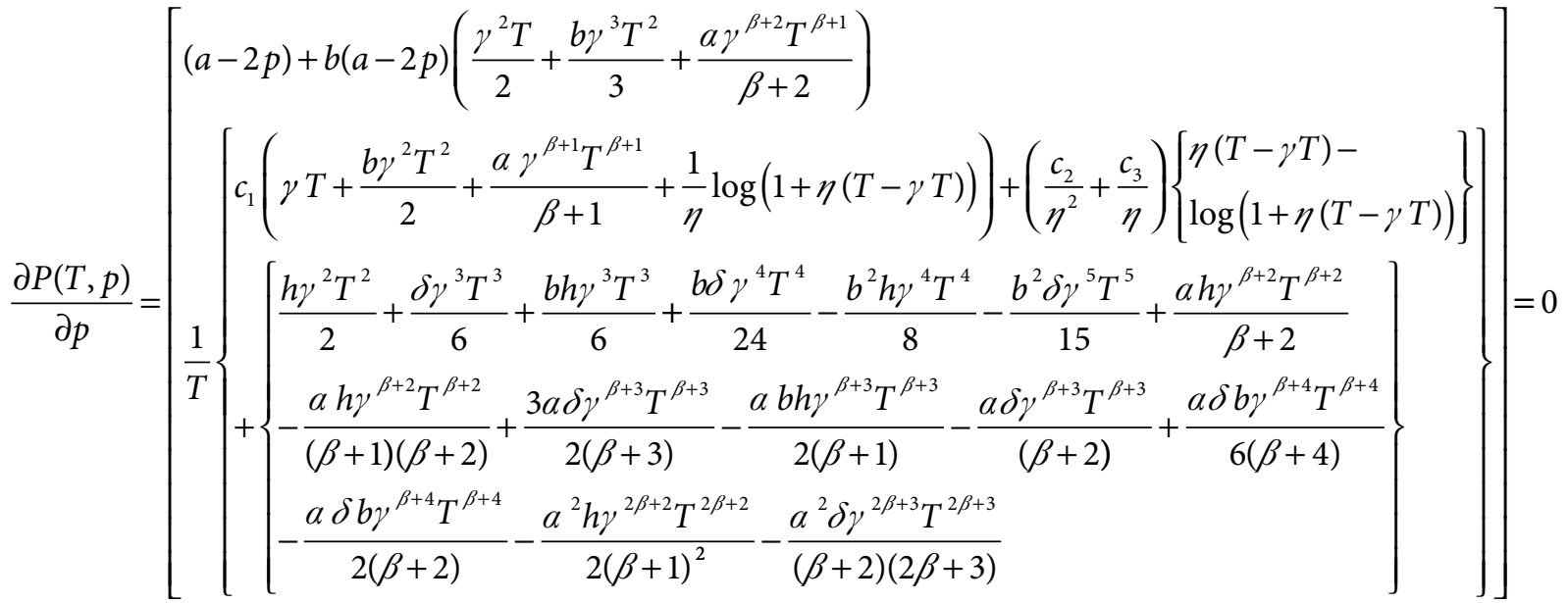

Using the software Mathematica-8.1, from equation (13) and (14) we can determine the optimum values of $T^{*}$ and $P^{*}$ simultaneously and the optimal value $P^{*}(T, p)$ of the average net profit is determined by (12) provided they satisfy the sufficiency conditions for maximizing $P^{\prime \prime}(T, p)$ are

$$
\frac{\partial^{2} P(T, p)}{\partial T^{2}}<0, \frac{\partial^{2} P(T, p)}{\partial p^{2}}<0 \text { and }
$$

$$
\frac{\partial^{2} P(T, p)}{\partial T^{2}} \frac{\partial^{2} P(T, p)}{\partial p^{2}}-\left(\frac{\partial^{2} P(T, p)}{\partial T \partial p}\right)^{2}>0
$$

\section{Numerical Example}

Let $A=250, a=180, b=0.015, c_{1}=20, c_{2}=5, c_{3}=25, \alpha=0.4$, $\beta=0.3, \gamma=0.5, h=0.6, \eta=0.5, \delta=0.04$ 


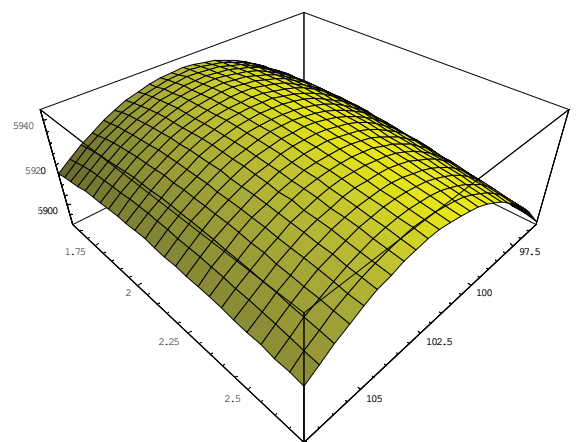

Figure 2. Concavity of the profit function.

Based on these input data, the findings are as follows:

$p^{*}=102.259, t_{1}^{*}=0.96327, Q^{*}=159.338, T^{*}=1.92654$ and $P^{*}(T, p)=5943.42$.

\section{Observations}

Some important inferences drawn from the Figures 3 to 11 are as follows:

(1) It is observe from the figures that optimal replenishment quantity and total profit increases as the parameters $a, b$ increases.

(2) As the parameter $c_{1}$ increases the order quantity increases but the total profit slightly decreases.

(3) The optimal replenishment quantity and total profit decreases as the parameters $c_{2}, \alpha, \eta$ and $h$ increases.

(4) The table shows that optimal order quantity decreases and the total profit increases as $\beta$ increases.

(5) The optimal order quantity and total profit very slightly decreases as the parameter $\delta$ increases.

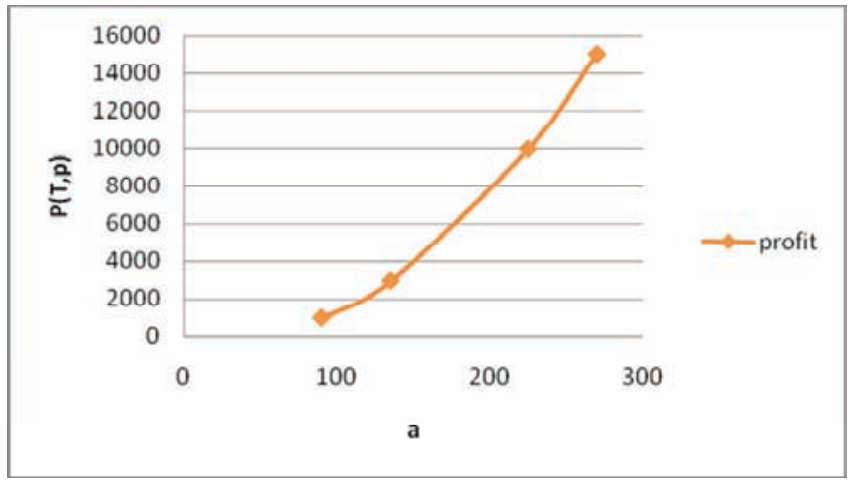

Figure 3. Net Profit v/s change in parameter a.

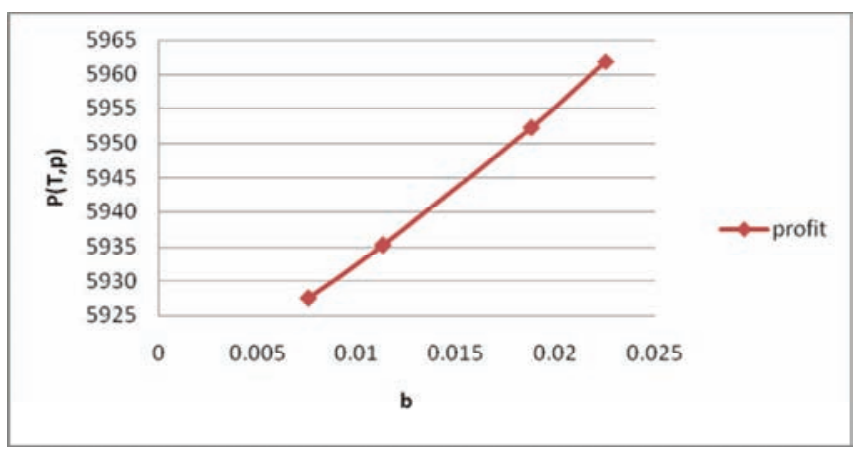

Figure 4. Net Profit v/s change in parameter b.

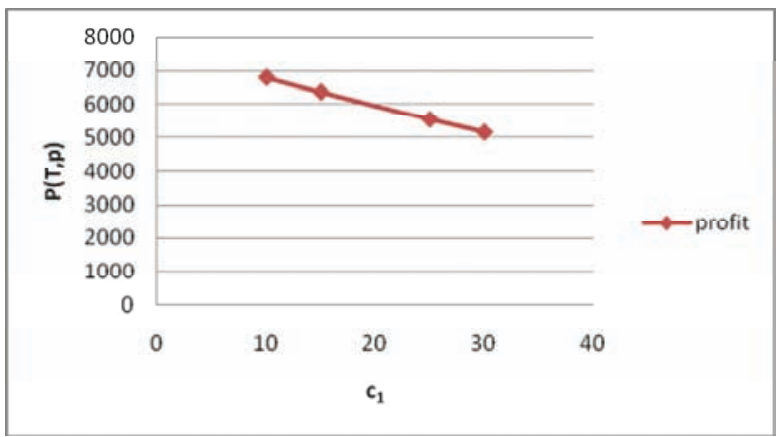

Figure 5. Net Profit v/s change in parameter $c_{1}$

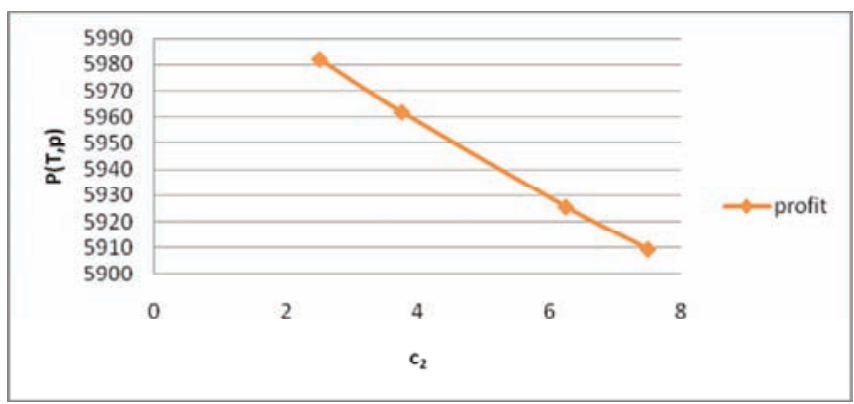

Figure 6. Net Profit v/s change in parameter $c_{2}$

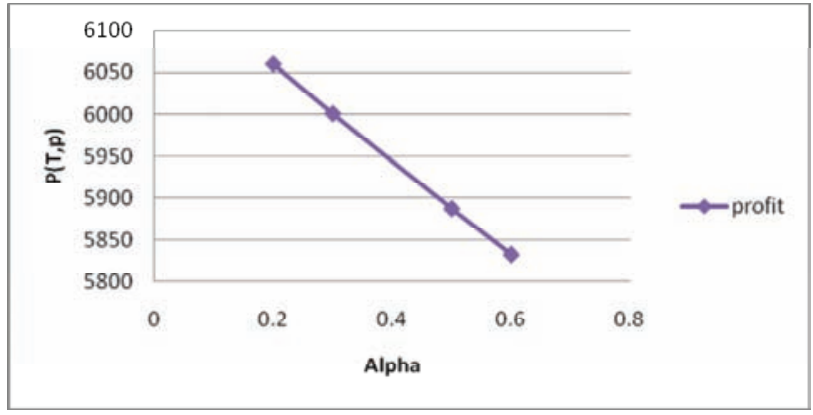

Figure 7. Net Profit v/s change in parameter $\alpha$. 


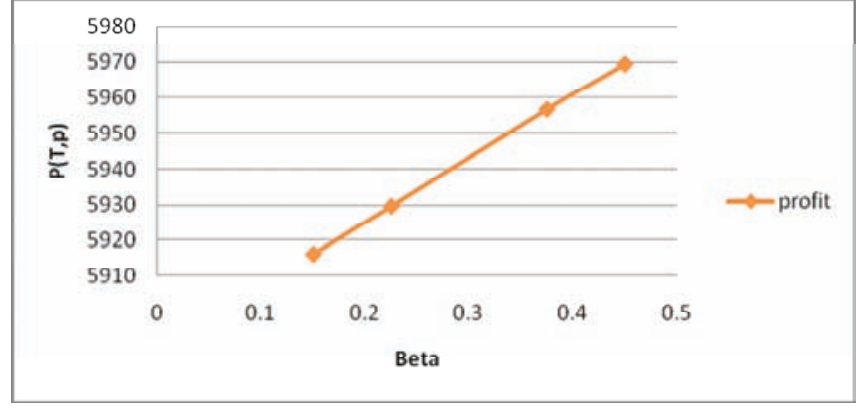

Figure 8. Net Profit v/s change in parameter $\beta$.

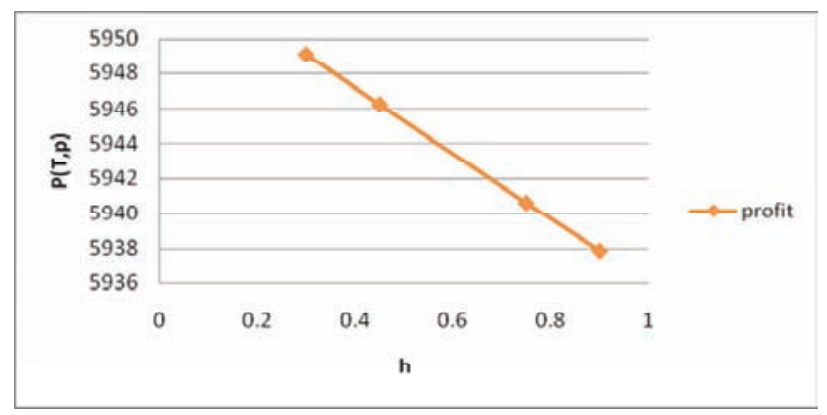

Figure 9. Net Profit v/s change in parameter h.

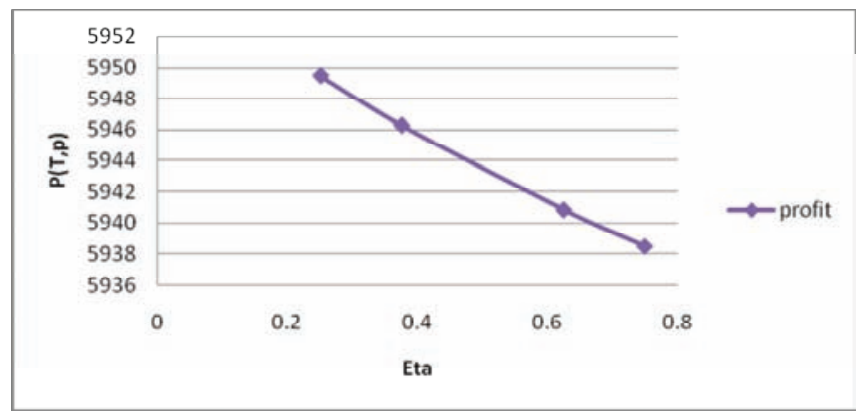

Figure 10. Net Profit v/s change in parameter $\eta$.

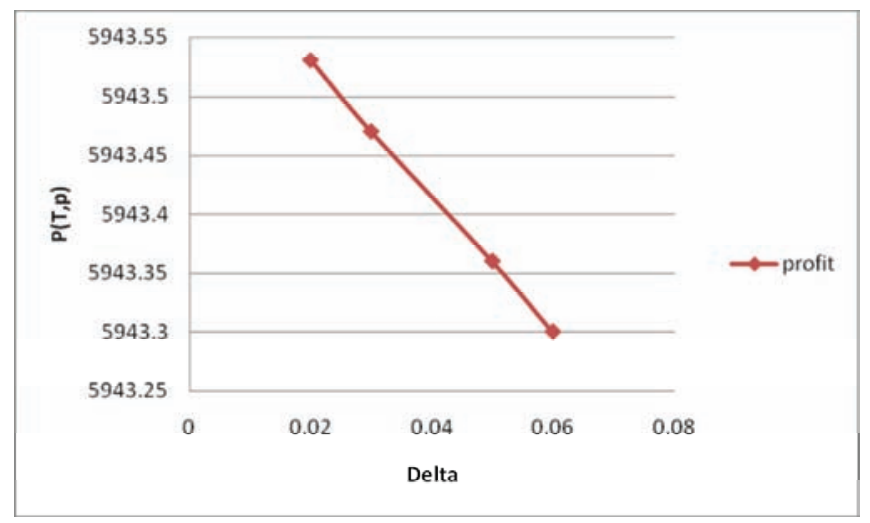

Figure 11. Net Profit v/s change in parameter $\delta$.

\section{Fuzzy Mathematical Model}

In this study we consider $a, A, c_{1}, c_{2}, c_{3}, h$ and $\delta$ as fuzzy numbers i.e, as $\hat{a}, \hat{A}, \hat{c}_{1}, \hat{c}_{2}, \hat{c}_{3}, \hat{h}$ and $\hat{\delta}$ Then $\mathrm{P}^{*}(\mathrm{~T}, \mathrm{P})$ is regarded as the estimate of total profit per unit time in the fuzzy sense.

$\hat{a}=\left(a-\Delta_{1}, \mathrm{a}, a+\Delta_{2}\right)$ where $0<\Delta_{1}<a$ and $\Delta_{1} \Delta_{2}>0$

$\widehat{A}=\left(A-\Delta_{3}, \mathrm{~A}, A+\Delta_{4}\right)$ where $0<\Delta_{3}<A$ and $\Delta_{3} \Delta_{4}>0$

$\hat{c}_{1}=\left(c_{1}-\Delta_{5}, c_{1}, c_{1}+\Delta_{6}\right)$ where $0<\Delta_{5}<c_{1}$ and $\Delta_{5} \Delta_{6}>0$

$\hat{c}_{2}=\left(c_{2}-\Delta_{7}, c_{2}, c_{2}+\Delta_{8}\right)$ where $0<\Delta_{7}<c_{2}$ and $\Delta_{7} \Delta_{8}>0$

$\hat{c}_{3}=\left(c_{3}-\Delta_{9}, c_{3}, c_{3}+\Delta_{10}\right)$ where $0<\Delta_{9}<c_{3}$ and $\Delta_{9} \Delta_{10}>0$

$\hat{h}=\left(h-\Delta_{11}, h, h+\Delta_{12}\right)$ where $0<\Delta_{11}<h$ and $\Delta_{11} \Delta_{12}>0$

$\hat{\delta}=\left(\delta-\Delta_{13}, \delta, \delta+\Delta_{14}\right)$ where $0<\Delta_{13}<\delta$ and $\Delta_{13} \Delta_{14}>0$

And the signed distance of $\hat{a}$ to $\hat{0}$ is given by the relation $\mathrm{d}(\hat{a}, \hat{0})=a+1 / 4\left(\Delta_{2}-\Delta_{1}\right)$ where $\mathrm{d}(\hat{a}, \hat{0})>0$ and $\mathrm{d}(\hat{a}, \hat{0})$ $\in\left[\mathrm{a}-\Delta_{1}, \mathrm{a}+\Delta_{2}\right]$

Similarly, the signed distance of other parameters to $\hat{0}$ is given by the relations

$\mathrm{d}(\widehat{A}, \hat{0})=A+1 / 4\left(\Delta_{4}-\Delta_{3}\right)$, where $\mathrm{d}(\widehat{A}, \hat{0})>0$ and $\mathrm{d}(\widehat{A}$,

$\hat{0}) \in\left[\mathrm{A}-\Delta_{3}, \mathrm{~A}+\Delta_{4}\right]$

$\mathrm{d}\left(\hat{c}_{1}, \hat{0}\right)=c_{1}+1 / 4\left(\Delta_{6}-\Delta_{5}\right)$, where $\mathrm{d}\left(\hat{c}_{1}, \hat{0}\right)>0$ and $\mathrm{d}\left(\hat{c}_{1}\right.$, $\hat{0}) \in\left[c_{1}-\Delta_{5}, c_{1}+\Delta_{6}\right]$

$\mathrm{d}\left(\hat{c}_{2}, \hat{0}\right)=c_{2}+1 / 4\left(\Delta_{8}-\Delta_{7}\right)$, where $\mathrm{d}\left(\hat{c}_{2}, \hat{0}\right)>0$ and $\mathrm{d}\left(\hat{c}_{2}\right.$, $\hat{0}) \in\left[\mathrm{c}_{2}-\Delta_{7}, \mathrm{c}_{2}+\Delta_{8}\right]$

$\mathrm{d}\left(\hat{c}_{3}, \hat{0}\right)=c_{3}+1 / 4\left(\Delta_{10}-\Delta_{9}\right)$, where $\mathrm{d}\left(\hat{c}_{3}, \hat{0}\right)>0$ and $\mathrm{d}\left(\hat{c}_{3}\right.$, $\hat{0}) \in\left[c_{3}-\Delta_{9}, c_{3}+\Delta_{10}\right]$

$\mathrm{d}(\hat{h}, \hat{0})=h+1 / 4\left(\Delta_{12}-\Delta_{11}\right)$, whered $(\hat{h}, \hat{0})>0$ and $\mathrm{d}(\hat{h}$, $\hat{0}) \in\left[\mathrm{h}-\Delta_{11}, \mathrm{~h}+\Delta_{12}\right]$

$\mathrm{d}(\hat{\delta}, \hat{0})=\delta+1 / 4\left(\Delta_{14}-\Delta_{13}\right)$, where $\mathrm{d}(\hat{\delta}, \hat{0})>0$ and $\mathrm{d}(\hat{\delta}$, $\hat{0}) \in\left[\mathrm{h}-\Delta_{13}, \mathrm{~h}+\Delta_{14}\right]$ 
Now, by the fuzzy triangular rule fuzzy total profit per unit is

$$
\operatorname{FP}\left(\hat{a}, \widehat{A}, \hat{c}_{1}, \hat{c}_{2}, \hat{c}_{3}, \hat{h}, \hat{\delta}\right)=\left(\mathrm{F}_{1}, \mathrm{~F}_{2}, \mathrm{~F}_{3}\right)
$$

And $\mathrm{F}_{1}, \mathrm{~F}_{2}, \mathrm{~F}_{3}$ are obtained as

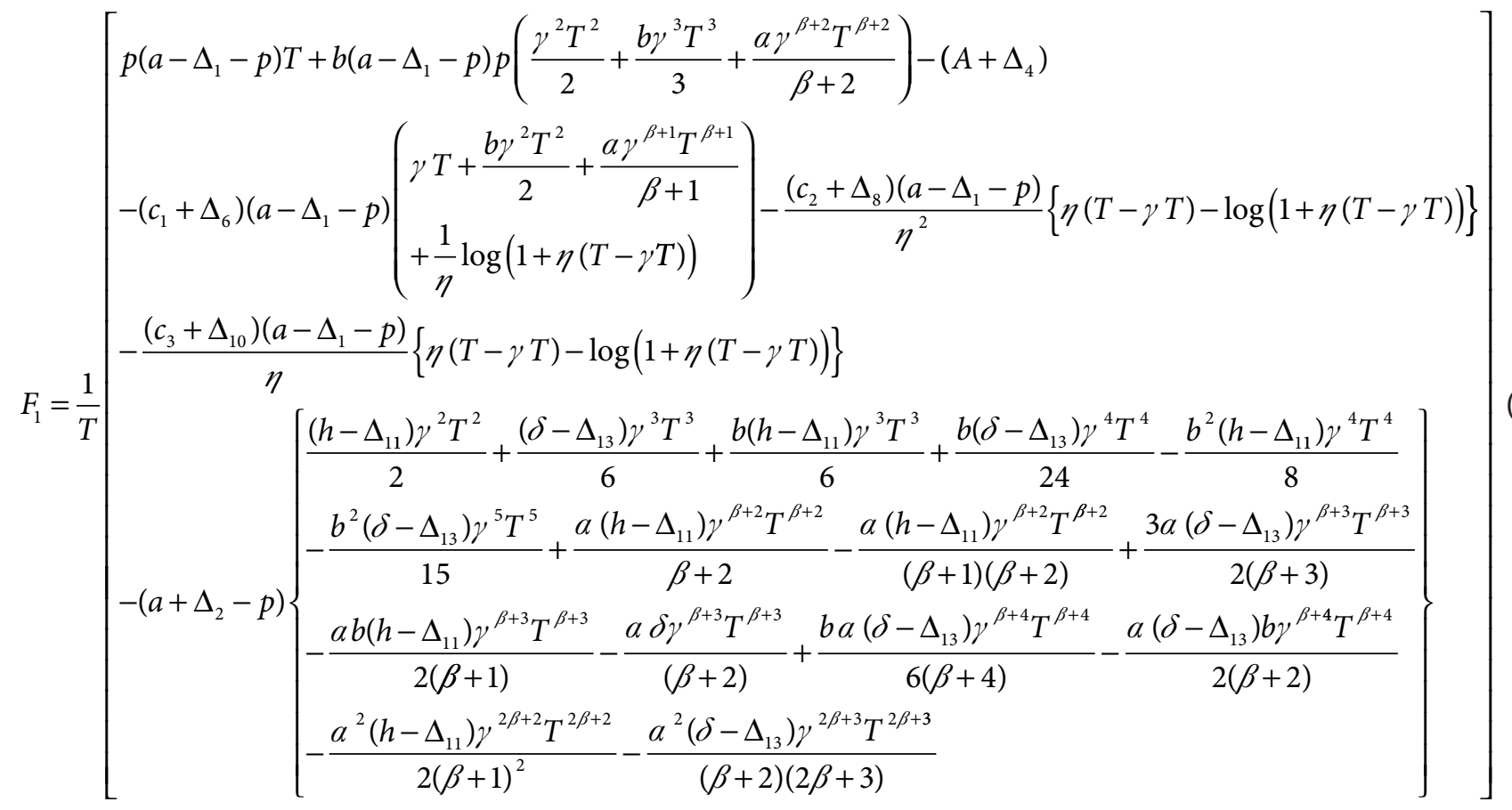

$$
F_{2}=\frac{1}{T}\left[\begin{array}{l}
p(a-p) T+b(a-p) p\left(\frac{\gamma^{2} T^{2}}{2}+\frac{b \gamma^{3} T^{3}}{3}+\frac{a \gamma^{\beta+2} T^{\beta+2}}{\beta+2}\right)-A \\
-c_{1}(a-p)\left[\begin{array}{l}
\left.\gamma T+\frac{b \gamma^{2} T^{2}}{2}+\frac{a \gamma^{\beta+1} T^{\beta+1}}{\beta+1}\right) \\
+\frac{1}{\eta} \log (1+\eta(T-\gamma T))
\end{array}\right)-\frac{c_{2}(a-p)}{\eta^{2}}\{\eta(T-\gamma T)-\log (1+\eta(T-\gamma T))\} \\
-\frac{c_{3}(a-p)}{\eta}\{\eta(T-\gamma T)-\log (1+\eta(T-\gamma T))\} \\
-(a-p)\left\{\begin{array}{l}
\frac{h \gamma^{2} T^{2}}{2}+\frac{\delta \gamma^{3} T^{3}}{6}+\frac{b h \gamma^{3} T^{3}}{6}+\frac{b \delta \gamma^{4} T^{4}}{24}-\frac{b^{2} h \gamma^{4} T^{4}}{8}-\frac{b^{2} \delta \gamma^{5} T^{5}}{15}+\frac{a h \gamma^{\beta+2} T^{\beta+2}}{\beta+2} \\
-\frac{a h \gamma^{\beta+2} T^{\beta+2}}{(\beta+1)(\beta+2)}+\frac{3 a \delta \gamma^{\beta+3} T^{\beta+3}}{2(\beta+3)}-\frac{a b h \gamma^{\beta+3} T^{\beta+3}}{2(\beta+1)}-\frac{a \delta \gamma^{\beta+3} T^{\beta+3}}{(\beta+2)}+\frac{a \delta b \gamma^{\beta+4} T^{\beta+4}}{6(\beta+4)} \\
-\frac{a \delta b \gamma^{\beta+4} T^{\beta+4}}{2(\beta+2)}-\frac{a^{2} h \gamma^{2 \beta+2} T^{2 \beta+2}}{2(\beta+1)^{2}}-\frac{a \gamma^{2} \delta \gamma^{2 \beta+3} T^{2 \beta+3}}{(\beta+2)(2 \beta+3)}
\end{array}\right.
\end{array}\right\}
$$




$$
F_{3}=\frac{1}{T}\left[\begin{array}{l}
p\left(a+\Delta_{2}-p\right) T+b\left(a+\Delta_{2}-p\right) p\left(\frac{\gamma^{2} T^{2}}{2}+\frac{b \gamma^{3} T^{3}}{3}+\frac{a \gamma^{\beta+2} T^{\beta+2}}{\beta+2}\right)-\left(A-\Delta_{3}\right) \\
-\left(c_{1}-\Delta_{5}\right)\left(a+\Delta_{2}-p\right)\left(\begin{array}{c}
\left.\gamma T+\frac{b \gamma^{2} T^{2}}{2}+\frac{a \gamma^{\beta+1} T^{\beta+1}}{\beta+1}\right) \\
+\frac{1}{\eta} \log (1+\eta(T-\gamma T))
\end{array}\right)-\frac{\left(c_{2}-\Delta_{7}\right)\left(a+\Delta_{2}-p\right)}{\eta^{2}}\{\eta(T-\gamma T)-\log (1+\eta(T-\gamma T))\} \\
-\frac{\left(c_{3}-\Delta_{9}\right)\left(a+\Delta_{2}-p\right)}{\eta}\{(T-\gamma T)-\log (1+\eta(T-\gamma T))\} \\
-\left(a-\Delta_{1}-p\right)\left\{\begin{array}{l}
\frac{\left(h+\Delta_{12}\right) \gamma^{2} T^{2}}{2}+\frac{\left(\delta+\Delta_{14}\right) \gamma^{3} T^{3}}{6}+\frac{b\left(h+\Delta_{12}\right) \gamma^{3} T^{3}}{6}+\frac{b\left(\delta+\Delta_{14}\right) \gamma^{4} T^{4}}{24}-\frac{b^{2}\left(h+\Delta_{12}\right) \gamma^{4} T^{4}}{8} \\
-\frac{b^{2}\left(\delta+\Delta_{14}\right) \gamma^{5} T^{5}}{15}+\frac{a\left(h+\Delta_{12}\right) \gamma^{\beta+2} T^{\beta+2}}{\beta+2}-\frac{a\left(h+\Delta_{12}\right) \gamma^{\beta+2} T^{\beta+2}}{(\beta+1)(\beta+2)}+\frac{3 a\left(\delta+\Delta_{14}\right) \gamma^{\beta+3} T^{\beta+3}}{2(\beta+3)} \\
-\frac{a b\left(h+\Delta_{12}\right) \gamma^{\beta+3} T^{\beta+3}}{2(\beta+1)}-\frac{a\left(\delta+\Delta_{14}\right) \gamma^{\beta+3} T^{\beta+3}}{(\beta+2)}+\frac{b a\left(\delta+\Delta_{14}\right) \gamma^{\beta+4} T^{\beta+4}}{6(\beta+4)}-\frac{a\left(\delta+\Delta_{14}\right) b \gamma^{\beta+4} T^{\beta+4}}{2(\beta+2)} \\
-\frac{a^{2}\left(h+\Delta_{12}\right) \gamma^{2 \beta+2} T^{2 \beta+2}}{2(\beta+1)^{2}}-\frac{a^{2}\left(\delta+\Delta_{14}\right) \gamma^{2 \beta+3} T^{2 \beta+3}}{(\beta+2)(2 \beta+3)}
\end{array}\right.
\end{array}\right]
$$

Now, defuzzified average profit is given by

$$
\tilde{P}(T, p)=\frac{F_{1}+2 F_{2}+F_{3}}{4}
$$

Also, the defuzzified order quantity is $\mathrm{Q}$ and is given by

$Q=\left(a+\frac{\left(\Delta_{2}-\Delta_{1}\right)}{4}-p\right)\left(t_{1}+\frac{b t_{1}^{2}}{2}+\frac{a t_{1}^{\beta+1}}{\beta+1}+\frac{1}{\eta} \log \left(1+\eta\left(T-t_{1}\right)\right)\right)$

The necessary conditions for maximizing the average profit are

$$
\frac{\partial \tilde{P}(T, p)}{\partial T}=0 \text { and } \frac{\partial \tilde{P}(T, p)}{\partial p}=0
$$

Using the software Mathematica-8.1, from the above two equations we can determine the optimum values of $\tilde{T}$ and $\tilde{p}$ simultaneously and the optimal value $\tilde{P}(T, p)$ of the average net profit is determined by (18).

\section{Numerical Example}

Let $\mathrm{A}=250, \Delta_{3}=12.5, \Delta_{4}=25, \mathrm{a}=180, \Delta_{1}=9, \Delta_{2}=18, \mathrm{~b}=$ $0.015, \mathrm{c}_{1}=20, \Delta_{5}=1, \Delta_{6}=2, \mathrm{c}_{2}=5, \Delta_{7}=0.25, \Delta_{8}=0.50, \mathrm{c}_{3}=$
$25, \Delta_{9}=1.25, \Delta_{10}=2.5, \alpha=0.4, \beta=0.3, \gamma=0.5, \mathrm{~h}=0.6, \Delta_{11}=$ $0.03, \Delta_{12}=0.06, \eta=0.5, \delta=0.04, \Delta_{13}=0.002, \Delta_{14}=0.004$.

Based on these input data, the findings are as follows:

$\mathrm{p}_{\mathrm{f}}^{*}=103.545, \mathrm{t}_{\mathrm{lf}}{ }^{*}=0.970155, \mathrm{Q}_{\mathrm{f}}{ }^{*}=162.444, \mathrm{~T}_{\mathrm{f}}^{*}=1.94031$ and $\tilde{P}(\mathrm{~T}, \mathrm{p})=6106.36$.

\section{Sensitivity Analysis}

Optimal solution for the model with fuzzy demand rate, ordering cost, holding cost, purchasing cost, shortage cost, opportunity cost.

\section{Observations}

(1) When $\left(\Delta_{1}, \Delta_{2}\right)$, varies, the optimal profit $\tilde{P}\left(\mathrm{p}_{\mathrm{f}}^{*}, \mathrm{~T}_{\mathrm{f}}^{*}\right)$ decreases.

(2) The optimal profit $\tilde{P}\left(\mathrm{p}_{\mathrm{f}}^{*}, \mathrm{~T}_{\mathrm{f}}^{*}\right)$ slightly increases when $\left(\Delta_{3}, \Delta_{4}\right),\left(\Delta_{7}, \Delta_{8}\right)$ and $\left(\Delta_{9}, \Delta_{10}\right)$ varies.

(3) When $\left(\Delta_{5}, \Delta_{6}\right)$, varies, the optimal profit $\tilde{P}\left(\mathrm{p}_{\mathrm{f}}^{*}, \mathrm{~T}_{\mathrm{f}}^{*}\right)$ increases.

(4) It is observed from the above table that there is a very small increment in profit $\tilde{P}\left(\mathrm{p}_{\mathrm{f}}^{*}, \mathrm{~T}_{\mathrm{f}}^{*}\right)$ as $\left(\Delta_{11}, \Delta_{12}\right)$ and $\left(\Delta_{13}, \Delta_{14}\right)$ varies. 


\section{Conclusion}

In the current study an inventory model is presented in which demand rate is considered as a function of price and stock both. In the development of model it is assumed that shortages are allowed and partially backlogged. The model is proposed in the following two senses: (1) crisp sense and (2) fuzzy sense. A numerical example to illustrate the problem in both the environments is provided and sensitivity analysis with respect to system parameters is also carried out.

In the present study a partially backlogged inventory model with stock and price sensitive demand is

\section{References}

1. Goswami A, and Chaudhuri K S (1991). An EOQ model for deteriorating items with shortages and a linear trend in demand, Journal of the Operational Research Society, vol 42 (12), 1105-1110.

2. Baker R C, and Urban T L (1988). A deterministic inventory system with an inventory level dependent demand rate, Journal of the Operational Research Society, vol 39(9), 823831.

3. Mandal B N, and Phaujdar S (1989). An inventory model for deteriorating items and Stock-dependent consumption rate, Journal of the Operational Research Society, vol 40(5), 483-488.

4. Chung K-J (2003). An algorithm for an inventory model with inventory-level dependent demand rate, Computers and Operations Research, vol 30(9), 1311-1317.

5. Chang C-T (2004). Inventory model with stock-dependent demand and nonlinear holding costs for deteriorating items, Asia-Pacific Journal of Operational Research, vol 21(4), 435-446.

6. Levin et al. (1972). Inventory models with stock- and pricedependent demand for deteriorating items based on limited shelf space, Yugoslav Journal of Operations Research, vol 20, No. $1,55-69$.

7. Alfares H K (2007). Inventory model with stock-level dependent demand rate and variable holding cost, International Journal of Production Economics, vol 108 (1-2), 259-265.

8. Park K S (1982). Inventory models with partial backorders, International Journal of Systems Science, vol 13(12), 1313-1317.

9. Hollier R H, and Mak K L (1983). Inventory replenishment policies for deteriorating items in a declining market, International Journal of Production Economics, vol 21(7), 813-826.

10. Goyal S K, and Giri B C (2003). The production-inventory problem of a product with time varying demand, production and deterioration rates, European Journal of Operational Research, vol 147(3), 549-557.

11. Wee H M (1995). A deterministic lot-size inventory model for deteriorating items with shortages and a declining market, Computers \& Operations Research, vol 22(3), 345-356.

12. Wu K S, Ouyang L Y et al. (2006). An optimal replenishment policy for non-instantaneous deteriorating items with stockdependent demand and partial backlogging, International Journal Production Economics, vol 101(2), 369-384.

13. Chang C T, Wu S J et al. (2009). Optimal payment time with deteriorating items under inflation and permissible delay in payments, International Journal of Systems Science, vol 40(10), 985-993.

14. Skouri K, Konstantaras I et al. (2009). Inventory models with ramp type demand rate, partial backlogging and Weibull deterioration rate, European Journal of Operational Research, vol 192(1), 79-92.

15. Goyal S K, and Chang C T (2009). Optimal ordering and transfer policy for an inventory with stock dependent demand, European Journal of Operational Research, vol 196(1), 177-185.

16. Chang C T, Chen Y-J et al. (2010). Inventory models with stock- and price dependent demand for deteriorating items based on limited shelf space, Yugoslav Journal of Operations Research, vol 20, No. 1, 55-69.

17. Singh S R, and Singh T J (2008). Perishable inventory model with quadratic demand, partial backlogging and permissible delay in payments, International Review of Pure and Applied Mathematics, vol 1, 53-66.

18. Zadeh L (1965). Fuzzy sets, Information and Control, vol 8(3), 338-353.

19. Park K S (1987). Fuzzy-set theoretic interpretation of economic order quantity, IEEE Transactions on Systems, Man and Cybernetics, vol 17(6), 1082-1084.

20. Xu R, and Zhai X (2010). Analysis of supply chain coordination under fuzzy demand in a two-stage supply chain, Applied Mathematical Modelling, vol 34(1), 129-139.

21. Ghare P M, and Schrader G P (1963). A model for an exponentially inventory, Journal of Industrial Engineering, vol $14,238-243$.

22. Covert R P, and Philip G C (1973). An EOQ model with Weibull deterioration, AIIE Transactions, vol 5, 323-326.

23. Raafat F (1991). Survey of literature on continuously deteriorating inventory models. Journal of the Operational Research Society, vol 40, 27-37.

24. Abdul and Murata (2011). Retailer's inventory policy for deteriorating items under partial trade credit policy, International Journal of Industrial Engineering Computations, vol 2 (2011), 699-714.

25. Giri and Chaudhuri (1998). A heuristic for replenishment of deteriorating items with time-varying demand and short- 
ages in all cycles, International Journal of Systems Science, vol 29(6), 551-555.

26. Gupta R, and Vrat (1986). Inventory model with multi-items under constraint systems for stock dependent consumption rate, Operations Research, vol 24, 41-42.

27. Teng et al. (2002). An optimal replenishment policy for deteriorating items with time-varying demand and par- tial backlogging, Operations Research Letters, vol 30(6), 387-393.

28. Chang et al. (1998). Optimization of fuzzy production inventory model with repairable defective products under crisp or fuzzy production, International Journal of Operations Research, vol 2, No.2, 31-37. 